\title{
Enterovirus D68 infection induces IL-17-dependent neutrophilic airway inflammation and hyperresponsiveness
}

Charu Rajput, ${ }^{1}$ Mingyuan Han, ${ }^{1}$ J. Kelley Bentley, ${ }^{1}$ Jing Lei, ${ }^{1}$ Tomoko Ishikawa, ${ }^{1}$ Qian Wu, ${ }^{1}$ Joanna L. Hinde, ${ }^{1}$ Amy P. Callear, ${ }^{2}$ Terri L. Stillwell, ${ }^{1}$ William T. Jackson, ${ }^{3}$ Emily T. Martin, ${ }^{2}$ and Marc B. Hershenson ${ }^{1,4}$

'Departments of Pediatrics and Communicable Diseases, University of Michigan Medical School, Ann Arbor, Michigan, USA. 2Department of Epidemiology, University of Michigan School of Public Health, Ann Arbor, Michigan, USA. ${ }^{3}$ Department of Microbiology and Immunology, University of Maryland School of Medicine, Baltimore, MD, USA. ${ }^{4}$ Department of Molecular and Integrative Physiology, University of Michigan Medical School, Ann Arbor, Michigan, USA.

Enterovirus D68 (EV-D68) shares biologic features with rhinovirus (RV). In 2014, a nationwide outbreak of EV-D68 was associated with severe asthma-like symptoms. We sought to develop a mouse model of EV-D68 infection and determine the mechanisms underlying airway disease. BALB/c mice were inoculated intranasally with EV-D68 (2014 isolate), RV-A1B, or sham, alone or in combination with anti-IL-17A or house dust mite (HDM) treatment. Like RV-A1B, lung EV-D68 viral RNA peaked 12 hours after infection. EV-D68 induced airway inflammation, expression of cytokines (TNF- $\alpha$, IL-6, IL-12b, IL-17A, CXCL1, CXCL2, CXCL10, and CCL2), and airway hyperresponsiveness, which were suppressed by anti-IL-17A antibody. Neutrophilic inflammation and airway responsiveness were significantly higher after EV-D68 compared with RV-A1B infection. Flow cytometry showed increased lineage-, NKp46-, ROR $\gamma \mathrm{t}^{+} \mathrm{IL}-17^{+} \mathrm{ILC} 3 \mathrm{~s}$ and $\gamma \delta \mathrm{T}$ cells in the lungs of EV-D68-treated mice compared with those in RV-treated mice. EV-D68 infection of HDM-exposed mice induced additive or synergistic increases in BAL neutrophils and eosinophils and expression of IL-17, CCL11, IL-5, and Muc5AC. Finally, patients from the 2014 epidemic period with EV-D68 showed significantly higher nasopharyngeal IL-17 mRNA levels compared with patients with RV-A infection. EV-D68 infection induces IL-17-dependent airway inflammation and hyperresponsiveness, which is greater than that generated by RV-A1B, consistent with the clinical picture of severe asthma-like symptoms.

Authorship note: ETM and MBH contributed equally to this work

Conflict of interest: The authors have declared that no conflict of interest exists.

Submitted: April 27, 2018

Accepted: July 3, 2018

Published: August 23, 2018

Reference information: JCI Insight. 2018;3(16):e121882. https://doi.org/10.1172/jici. insight.121882.

\section{Introduction}

Enterovirus D68 (EV-D68) is a nonpolio human enterovirus that shares some biologic features with human rhinoviruses (RVs) (1). Human RV-87 was subsequently identified as EV-D68 by molecular diagnosis (2). First isolated in California in 1962 (3) and once considered rare, EV-D68 infection has been on a worldwide upswing in the 21st century. Six clusters (each equal to 10 or more cases) between 2005 and 2011 were reported from the Philippines, Japan, the Netherlands, and the United States (4). However, in 2014, the United States experienced a nationwide outbreak of EV-D68 associated with severe respiratory illness (5). From mid-August 2014 to January 15, 2015, the Center for Disease Control and state public health laboratories confirmed a total of 1,153 people with respiratory illness caused by EV-D68 (6). The 2014 outbreak was also associated with a rare polio-like illness in which patients suffered muscle weakness or paralysis from which there was poor motor recovery (7). Shortly afterward, the outbreak spread to Canada, Europe, and Asia (8).

In an epidemiologic study of EV-D68-infected hospitalized patients from the United States, the median age was 5 years (9). Respiratory symptoms commonly included dyspnea, cough, wheeze, and fever. The majority of patients were admitted to intensive care units, and over one-quarter received ventilator support. Over half the patients had a history of asthma or reactive airway disease, and patients with a history of asthma or reactive airway disease were significantly more likely to require intensive care admission and ventilator support $(9,10)$. Compared with RV-infected children, EV-D68 ${ }^{+}$patients were more likely to have a history of asthma and present with acute respiratory illness, especially status asthmaticus (11). 


\section{A}
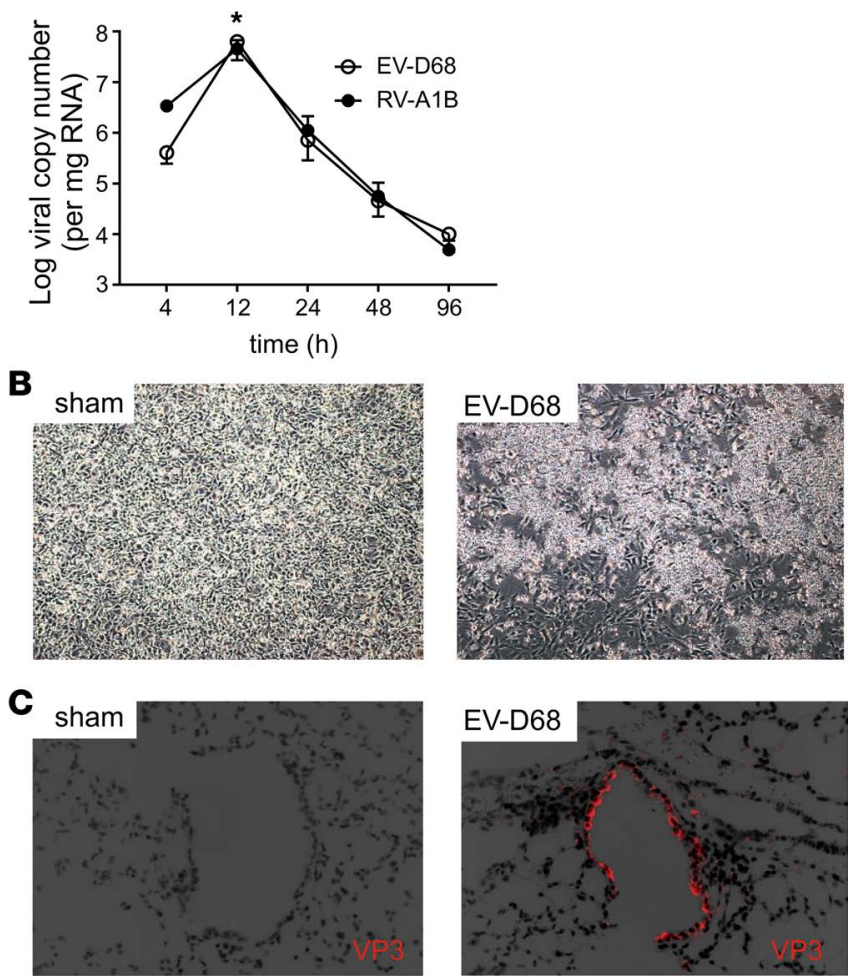

Figure 1. Viral RNA is detectable in the lungs of EV-D68-treated mice. (A) Female 8- to 10-week-old BALB/C mice were inoculated with $5 \times 10^{6} \mathrm{ePFU}$ of EV-D68 or $5 \times 10^{6} \mathrm{PFU}$ of RV-A1B by intranasal instillation, and lungs and nasal washes were examined by RT-PCR for viral RNA at the indicated time points. There was a small significant increase in lung viral RNA copy number between the 4- and 12-hour time point, and RNA was detected up to day 4. Data are shown as mean \pm SEM; $n=3$ mice/group from 3 different experiments; ${ }^{*} P<0.05$ by 1 -way ANOVA, compared with 4 -hour time point. (B) Supernatants from homogenized mouse lungs from sham- or EV-D68-inoculated mice were overlaid onto confluent rhabdomyosarcoma cell monolayers and examined for cytopathic effects. The image on the right shows cytopathic effects of EV-D68. (C) After inoculation, lungs were fixed and processed for immunofluorescence staining using Alexa Fluor 568-labeled anti-VP3 antibody. The image on the right shows EV-D68 in epithelial cells. Images were taken at $\times 200$ magnification.

The mechanisms by which EV-D68 promotes severe respiratory illness are unknown. Worse clinical outcomes associated with influenza are thought to be due in part to an exaggerated inflammatory response to viral infection (12). We therefore hypothesized that the 2014 EV-D68 epidemic was associated with more severe respiratory outcomes than RV due to differences in the host response to viral infection. To study the underlying mechanisms, we developed a model of EV-D68 infection in mature mice, comparing inflammatory responses to those induced by RV-A1B. We also compared the response of allergen-sensitized and -challenged mice to the two viruses.

\section{Results}

EV-D68 is detected in lungs up to day 4 after infection. Female BALB/c mice were anesthetized and inoculated with an epidemic strain of EV-D68 (50 $\mu 1$ at $10^{8} \mathrm{PFU}$ equivalents [ePFU]/ml) or RV-A1B by intranasal instillation. Mice were sacrificed, and lungs were analyzed by RT-PCR at 4-, 12-, 24-, 48-, and 96-hour time intervals for the presence of viral RNA. Viral RNA was detected after EV-D68 and RV-A1B inoculation (Figure 1A). We detected similar levels of viral replication for the two viruses 12 hours after inoculation. Thereafter, viral copy numbers gradually declined and minimal viral RNA was detected 4 days after the initial inoculation.

We also homogenized lungs 1-4 days after exposure and overlaid clarified supernatant containing EV-D68 on confluent rhabdomyosarcoma cell monolayers. The cells were examined for cytopathic effect, and cell lysates were examined for viral RNA by real-time PCR. Lung homogenate from EV-D68-infected mice induced cytopathic effects (Figure 1B), and viral RNA was detectable in rhabdomyosarcoma cell lysates (Table 1). 
Table 1. Effects of lung homogenates from EV-D68-infected mice on rhabdomyosarcoma cell monolayers

\begin{tabular}{cccc}
\hline Lung homogenates & Cytopathic effects & EV-D68 RNA (cycle no.) & 18s RNA (cycle no.) \\
Sham & - & 37.65 & 8.12 \\
EV-D68-4h & ++ & 6.70 & 8.43 \\
EV-D68-12h & +++ & 4.21 & 8.21 \\
EV-D68-24h & ++ & 6.56 & 8.54 \\
EV-D68-48h & + & 28.29 & 8.06
\end{tabular}

Cells were examined for cytopathic effects and viral RNA 48 hours after treatment with lung homogenates. - and + refer to the degree of cytopathic effects, with - indicating no cytopathic effects and +++ indicating severe cytopathic effects.

Lungs were also formalin-fixed and paraffin-embedded 24 hours after exposure, and sections were stained for EV-D68 immunofluorescence. Specific staining was seen in the airway epithelial cells of mice inoculated with EV-D68 but not in those of mice treated with an equal volume of sham (Figure 1C).

EV-D68 induces airway inflammation in lungs of naive mice. We examined the time course of airway inflammation after exposure to EV-D68. Mice were exposed to sham or EV-D68 for 1, 2, 4, and 7 days, and lungs were analyzed for bronchoalveolar lavage (BAL) analysis, H\&E staining, and qPCR. One and two days after infection, EV-D68-infected mice showed a significant increase in BAL monocytes, neutrophils, and lymphocytes compared with sham-infected animals (Figure 2A). On day 4, the BAL cell numbers returned toward baseline. EV-D68-infected mice also demonstrated significant increases in lung IFN- $\gamma,-\alpha$, and $-\beta$ mRNA expression (Figure 2B), consistent with the presence of viral RNA. We observed a similar pattern of IFN induction in RV-A1B-infected mice (13). The mRNA expression of CXCL10, CXCL1, CXCL2, CCL2, TNF- $\alpha$, and IL-12b was increased after EV-D68 exposure compared with that in sham controls (Figure 2B). Cytokine mRNA expression was maintained 24 and 48 hours after infection and decreased thereafter.

$E V$-D68 induces greater neutrophil recruitment and airway responsiveness than $R V$-A1B. Formalin-fixed, paraffin-embedded lungs harvested 48 hours after inoculation were stained with H\&E. Sham-inoculated mice showed no inflammation (Figure 3A). Both EV-D68- and RV-A1B-exposed mice showed leukocyte infiltration around large airways, which appeared to be greater in case of EV-D68 (Figure 3A). We determined BAL inflammatory cell counts in EV-D68- and RV-A1B-infected mice 48 hours after treatment. EV-D68-infected mice had significantly greater neutrophil recruitment than RV-A1B-treated mice (Figure 3B). The cell numbers of monocytes, lymphocytes, and eosinophils did not vary between the two groups. qPCR analysis showed significantly greater mRNA expression of IL-17A and its target genes, CXCL1 and CXCL2 (Figure 3C), each of which play a role in neutrophil chemotaxis (14, 15). Expression of IL-17F, a closely related IL-17 family member with proinflammatory effects (16); CXCL5, another CXCR2 ligand induced by viral infection (17); IFN- $\gamma$; and CXCL10 was comparable between EV-D68 and RV-A1B (Figure 3C). IL-17A protein abundance was also significantly higher in the lungs of EV-D68-infected mice (Figure 3D).

We also determined airway responsiveness in sham-, EV-D68-, RV-A1B- treated mice. Two days after infection, increasing doses of nebulized methacholine were given by inhalation, and respiratory system resistance was measured. EV-D68-infected mice demonstrated a significant increase in cholinergic airway responsiveness compared with RV-A1B- and sham-treated mice (Figure 3E).

We performed flow cytometry to determine the cellular source of IL-17A. Focusing on innate immune cells, we examined lung cells for lineage ${ }^{+} \mathrm{ROR} \gamma \mathrm{t}^{+} \gamma \delta \mathrm{T}$ cells and lineage-ROR $\gamma \mathrm{t}^{+} \mathrm{NKp} 46^{-}$type 3 innate lymphoid cells (ILC3s), each of which produce IL-17 (18-31). To test this, we performed flow cytometric analysis on sham-treated, EV-D68-infected, and RV-A1B-infected mice. EV-D68-infected mice showed a greater percentage of IL-17+ lung cells (Figure 4A). Cells were then sorted into lineage ${ }^{+}$ and lineage ${ }^{-}$cells (Figure 4B). EV-D68-infected mice showed a significant increase in lineage-NKp46ROR $\gamma \mathrm{t}^{+}$cells (Figure 4C). NKp46-ROR $\gamma \mathrm{t}^{+}$cells stained positive for IL-17 (Figure 4C). Both EVD-68 and RV-A1B infection increased the number of lineage ${ }^{+} \mathrm{TCR} \gamma \delta^{+} \mathrm{ROR} \gamma \mathrm{t}^{+}$cells (Figure 4D), indicating the presence of $\gamma \delta$ T cells. Further analysis demonstrated that $\gamma \delta$ T cells stained positive for IL-17 (Figure 4D). Together, these data show that differential neutrophilic inflammation in EV-D68-infected mouse lungs is associated with lineage- $\mathrm{ROR} \gamma \mathrm{t}^{+} \mathrm{NKp} 46^{-}$ILC3s. 
A
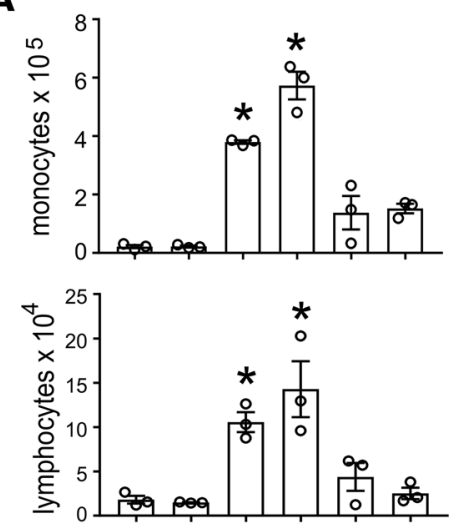

time (d) $\frac{1 \quad 2}{\text { sham }} \frac{1 \quad 2 \quad 4 \quad 7}{\text { EV-D68 }}$
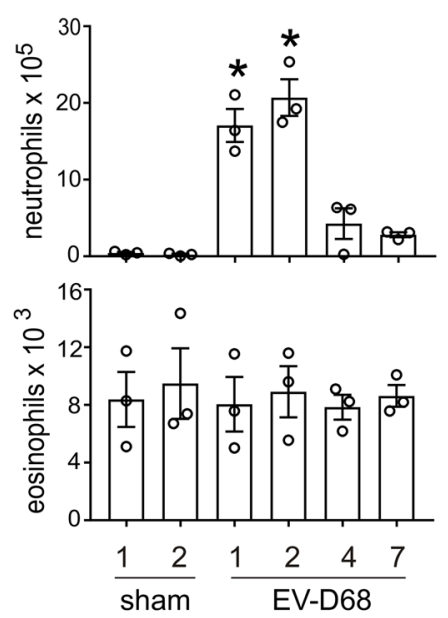

B

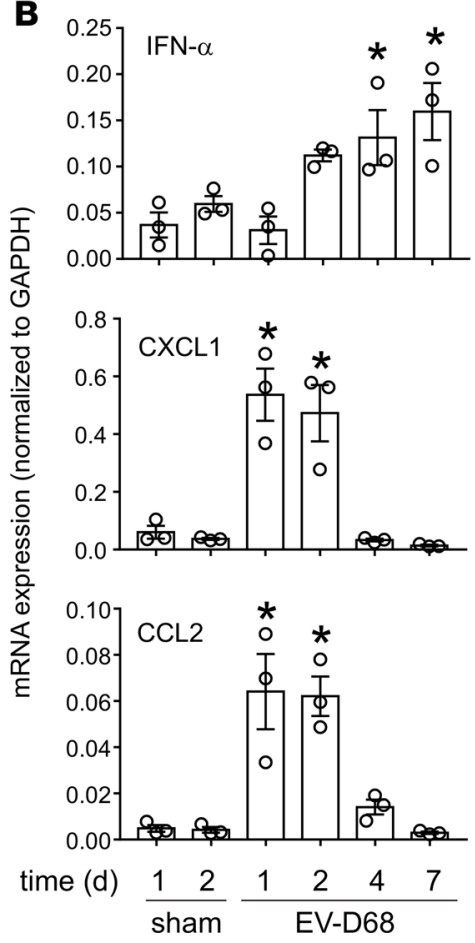

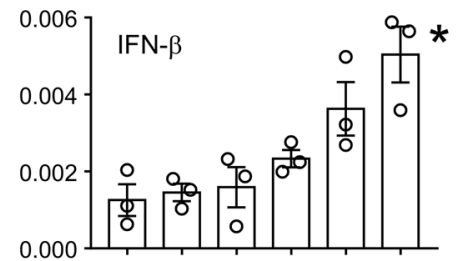
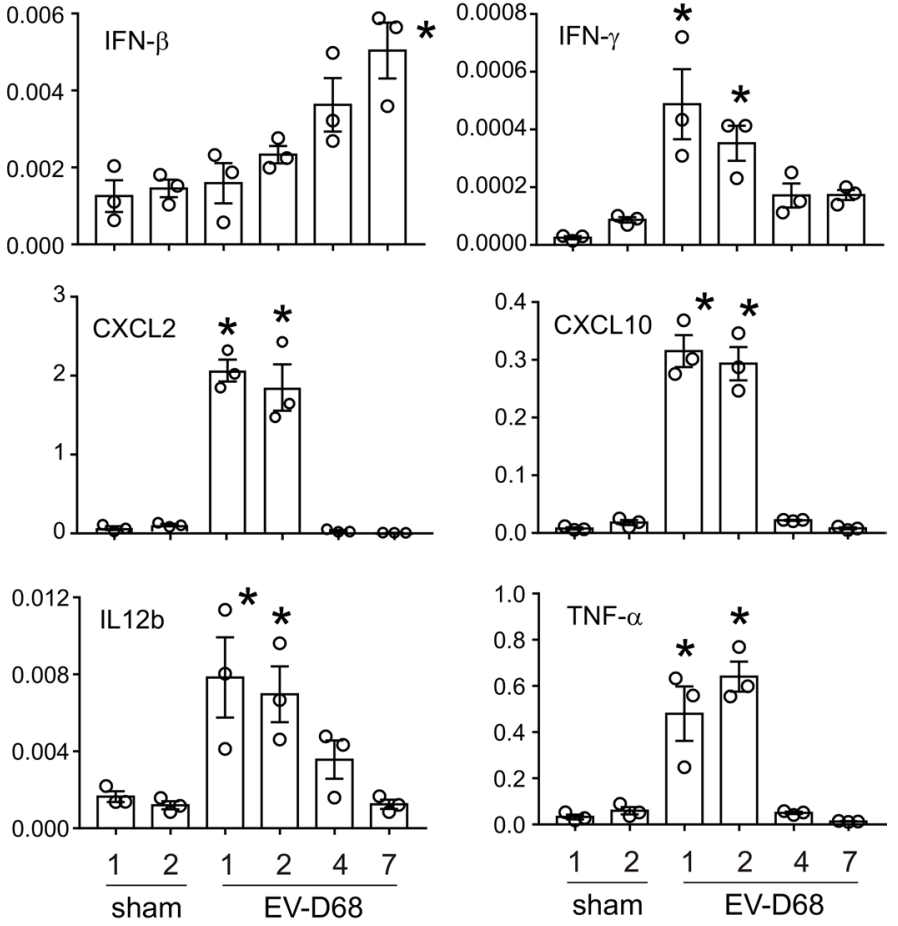

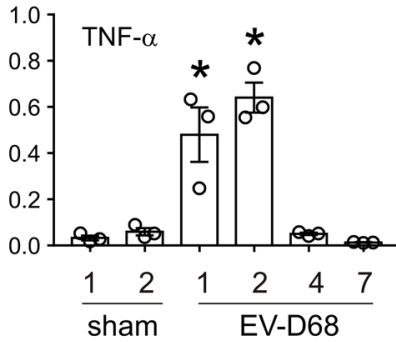

Figure 2. EV-D68 inoculation increases airway inflammation. Female 8- to 10 -week-old BALB/c mice were treated with sham or $5 \times 10^{6}$ ePFU of EV-D68. The lungs were harvested after the indicated time points and processed for BAL and mRNA expression. (A) Inflammatory cell counts in BAL at indicated time points. (B) mRNA expression analysis of indicated genes at indicated time points. Data are shown as mean $\pm \mathrm{SEM} ; n=3$ mice in each group from two different experiments; ${ }^{*} P<0.05$ by 1-way ANOVA, compared with sham.

Anti-IL-17 suppresses EV-D68-induced airway hyperresponsiveness. We determined whether IL-17 is required for EV-D68-induced airway inflammation and hyperresponsiveness. Mice were treated with anti-IL-17 or isotype control IgG and inoculated with sham, EV-D68, or RV-A1B 1 hour later. After 48 hours, BAL cell counts and airway responsiveness were determined. Mice infected with EV-D68 and pretreated with antiIL-17 showed lower neutrophil infiltration compared with mice pretreated with IgG (Figure 5A). There was no significant decrease in neutrophil infiltration in RV-A1B-infected mice. Anti-IL-17 pretreatment significantly reduced mRNA expression of CXCL1, CXCL2, CXCL10, and IFN- $\gamma$ in EV-D68- but not RV-1B-infected mice (Figure 5B). mRNA expression of TNF- $\alpha$ and CCL2 was not affected by anti-IL-17 pretreatment (Figure 5B). We also determined airway responsiveness in the different treatment groups. EV-D68-infected mice pretreated with anti-IL-17 antibody showed lower airway responsiveness compared with IgG-pretreated mice (Figure 5C). However, RV-A1B-infected mice did not show a similar decrease. 
A

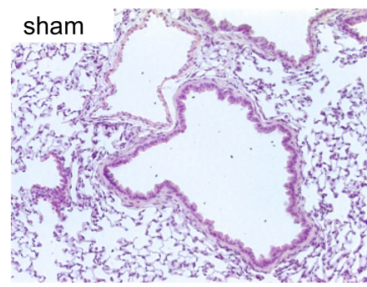

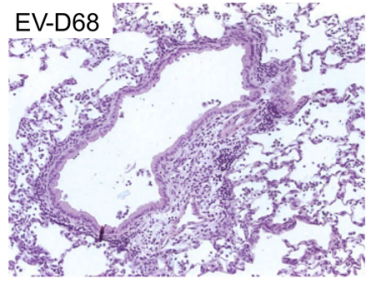

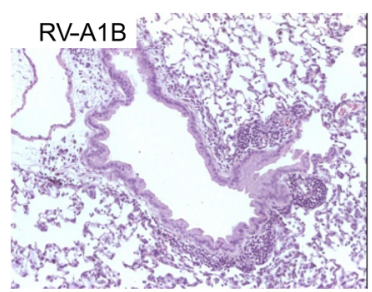

B
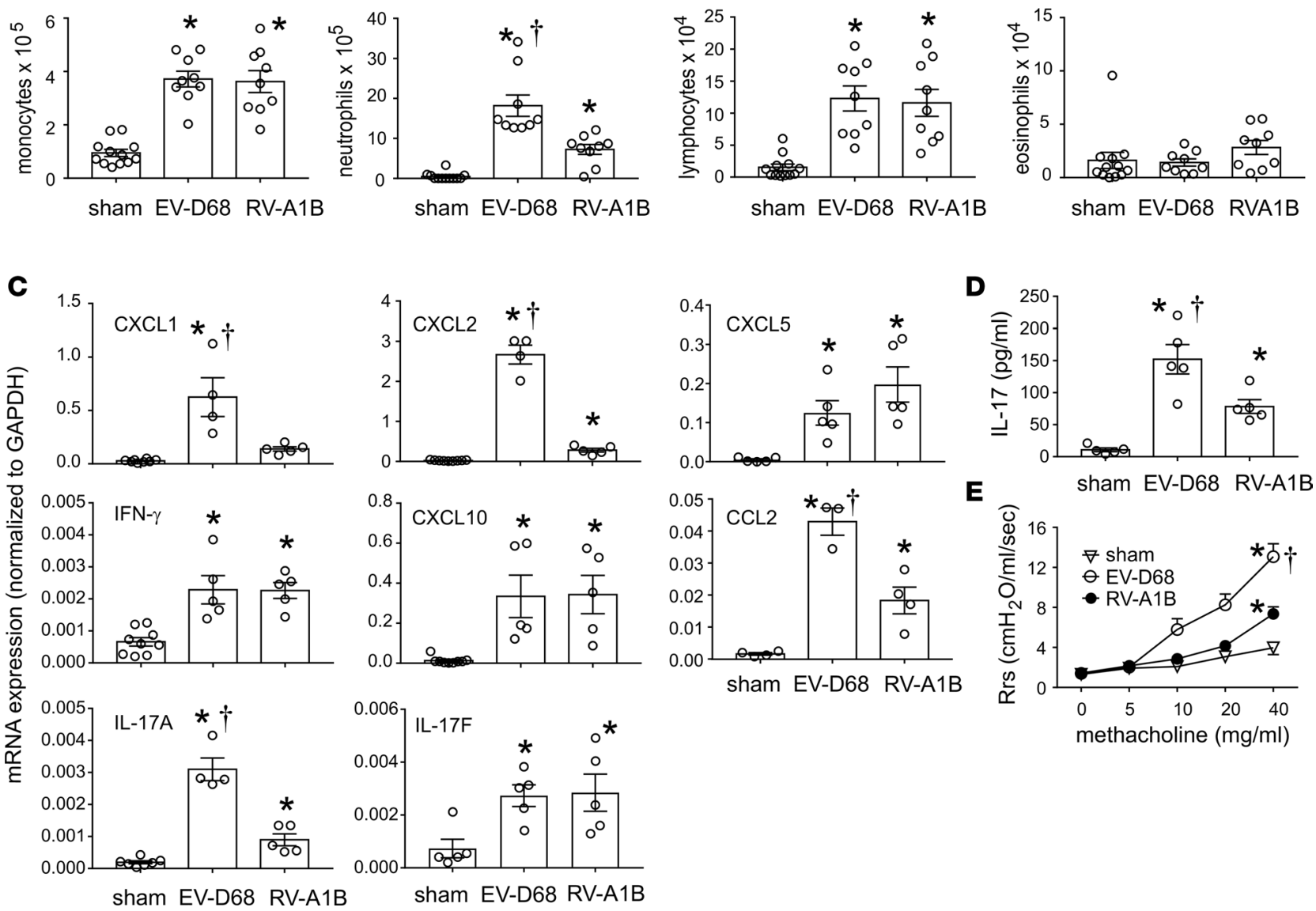

Figure 3. EV-D68 induces greater neutrophil inflammation than RV-A1B. Female 8 - to 10 -week-old BALB/c mice were treated with sham, $5 \times 10^{6}$ ePFU of EV-D68, or $5 \times 10^{6}$ PFU of RV-A1B. Mice were sacrificed 48 hours later and analyzed for histology, BAL, and qPCR. In another set of mice, changes in airway resistance to nebulized increasing doses of methacholine ( 0 to $40 \mathrm{mg} / \mathrm{ml}$ ) were assessed. (A) Images showing the inflammation in lungs 48 hours after treatment with sham, EV-D68, or RV-A1B. Images were taken at $\times 200$ magnification. (B) Analysis of BAL inflammatory cells. (C) qPCR analysis of indicated genes. (D) BAL IL-17 protein levels in the indicated groups. (E) Airway responsiveness to methacholine in the 3 groups of mice. Data are shown as mean \pm SEM of 3-5 mice/group from at least 2 different experiments; ${ }^{*} P<0.05$ by 1 -way ANOVA, compared with sham; $†<<$ 0.05 by $1-$ way ANOVA, compared with RV-1B.

EV-D68 inoculation enhances $m R N A$ expression of type 2 cytokines, mucus genes, and airway hyperresponsiveness in allergen-challenged mice. Most hospitalized cases of EV-D68-induced respiratory illness are associated with a previous history of asthma (9). We therefore determined the response to EV-D68 infection in mice with allergic airway disease. We exposed mice to HDM for up to 11 days followed by inoculation with sham, EV-D68, or RV-1B. Forty-eight hours later, mice were sacrificed for BAL and lung mRNA determination or anesthetized and endotracheally intubated for measurement of methacholine responsiveness. HDM sensitization and challenge induced lung infiltration with monocytes, lymphocytes, and eosinophils (Figure 6A). Allergen challenge induced lung mRNA expression of the eosinophil chemoattractants, CCL11 and CCL24; the type 2 cytokines, IL-5 and IL-13; and the mucus-related genes, Gob5 and Muc5AC (Figure 6B). As noted previously, EV-D68 infection increased BAL monocytes, neutrophils, and lymphocytes, while inducing mRNA expression of IFN- $\gamma$, CXCL10, and IL-17A. The combined effects of HDM 
A

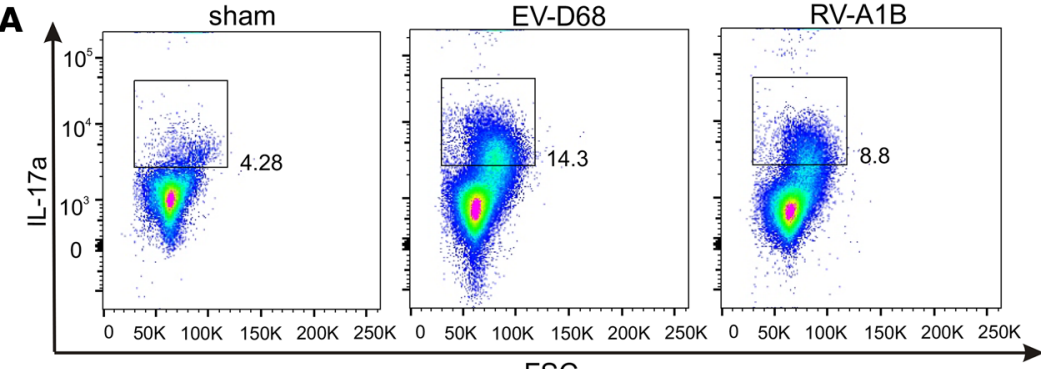

B

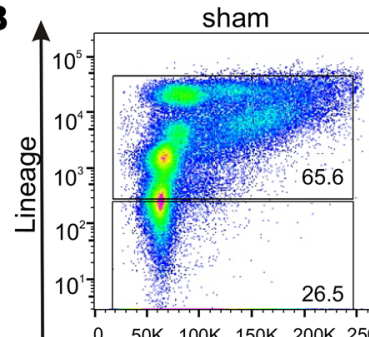

EV-D68
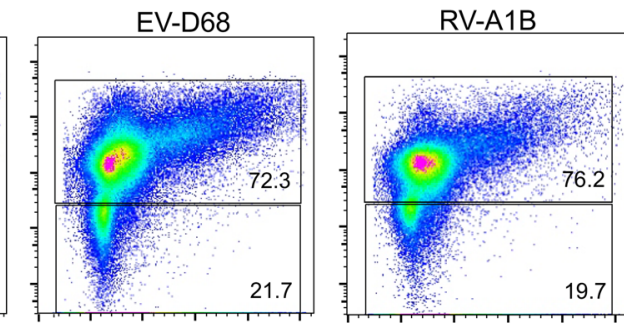

FSC
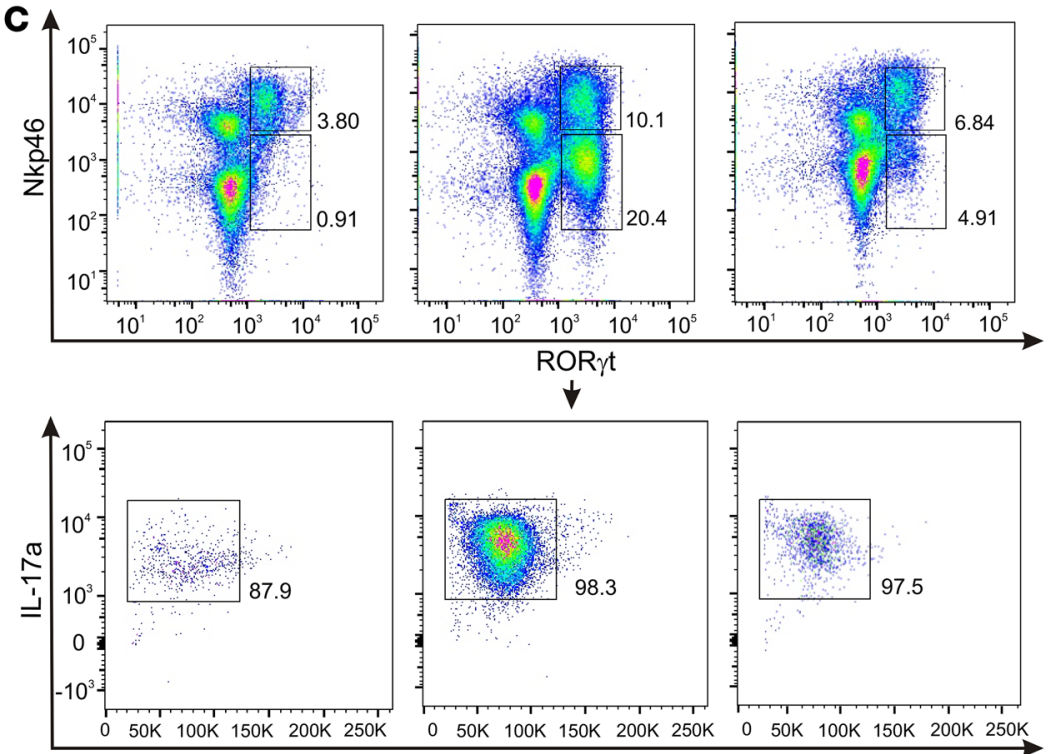

FSC

\section{D}
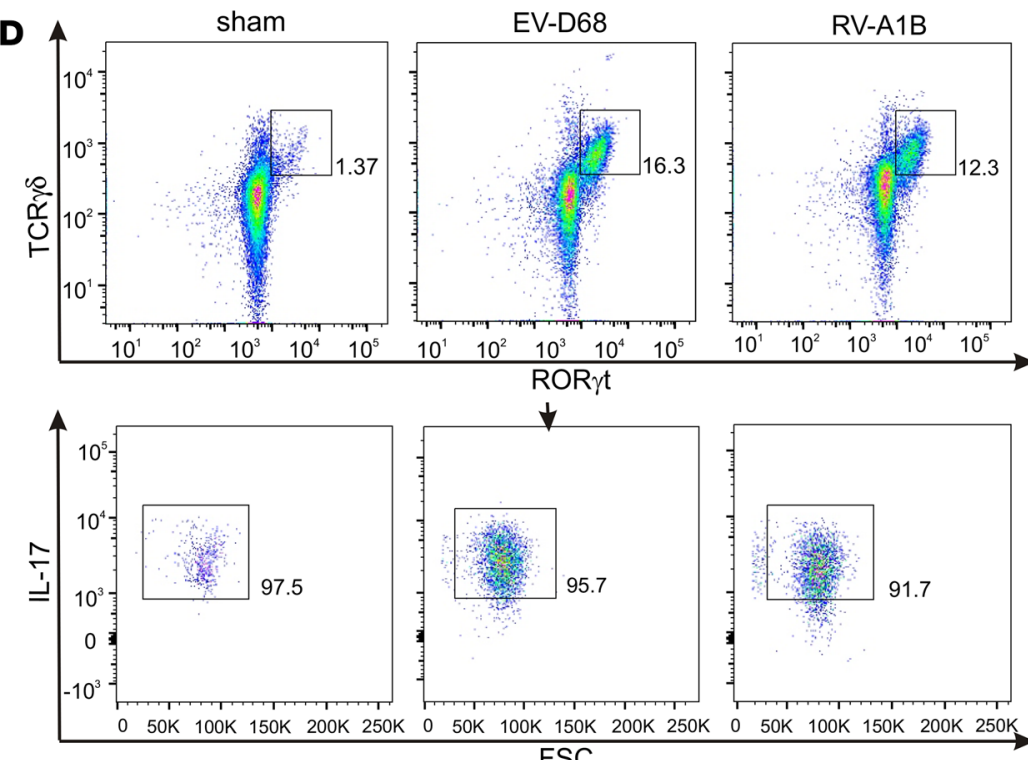
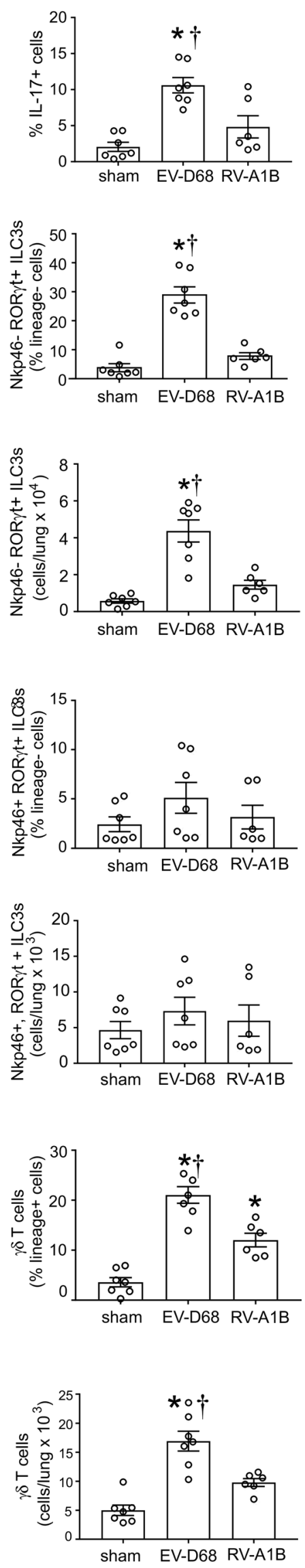
Figure 4. Flow cytometric analysis of IL-17-producing lung cells. Female 8 - to 10 -week-old BALB/c mice were treated with sham, $5 \times 10^{6}$ ePFU of EV-D68, or $5 \times 10^{6} \mathrm{PFU}$ of RV-A1B. Forty-eight hours later, lungs were harvested, digested with collagenase, and stained with lineage antibody cocktail, anti-NKp46, anti-TCR $\gamma \delta$, anti-ROR $\gamma \mathrm{t}$, anti-IL-17, and Pacific Blue (for dead cells). Cells were washed, fixed, and processed for flow cytometry. (A) Flow cytometry of live IL-17+ cells in sham, EV-D68, and RV-A1B groups. Group mean data are also shown. (B) Dot blots of lineage ${ }^{+}$and lineage- live cells for the 3 conditions. (C) NKp46 and ROR $\gamma$ t staining of lineage- cells. Group mean data for NKp46-, ROR $\gamma \mathrm{t}^{+}$, and NKp46 and ROR $\gamma \mathrm{t}$ double-positive cells are also shown. NKp46and ROR $\gamma \mathrm{t}^{+}$double-positive cells are gated for IL-17 staining. (D) TCR $\gamma \delta$ and ROR $\gamma \mathrm{t}$ staining of lineage ${ }^{+}$cells. Group mean data are also shown. TCR $\gamma \delta$ and ROR $\gamma$ t double-positive cells are gated for IL-17 staining. Data are shown as mean \pm SEM of 6-7 mice/group from 2 separate experiments; ${ }^{*} P<0.05$ by 1-way ANOVA, compared with sham; $\uparrow P<0.05$ by 1-way ANOVA, compared with RV-1B.

and EV-D68 were complex. EV-D68 infection of mice with allergic airway disease had additive effects on BAL eosinophils and lung IL-5, IL-13, CCL11, and Muc5AC mRNA expression (Figure 6, A and B). Thus, EV-D68 infection enhanced allergen-induced type 2 inflammation. EV-D68 infection of HDM-exposed mice also increased airway neutrophils and lung mRNA expression of IFN- $\gamma$, CXCL10, and IL-17A. However, these type 1 responses were significantly lower in allergen-challenged mice, consistent with the notion that prior allergen exposure dampens the antiviral response.

Next, we examined the effects of EV-D68 infection on the airway responsiveness of HDM-sensitized and -challenged mice. As expected, allergen-challenged mice showed increased airway responsiveness compared with PBS-treated mice (Figure 6C). However, EV-D68 infection of HDM-treated mice further induced airway resistance when compared with mice challenged with allergen or infected with EV-D68 alone.

$m R N A$ expression from nasopharyngeal swabs of patients with respiratory viral infections. At the University of Michigan, there was a spike in respiratory illnesses in September 2014. Patients suspected of having a respiratory virus were tested using the FilmArray Respiratory Panel (Biofire Diagnostics). RV/EV ${ }^{+}$samples were archived at our request, and RNA was extracted for future analysis. Nasopharyngeal samples from 10 patients with EV-D68 infection and 11 patients with RV-A infection were examined for IL-17 mRNA by qPCR. Demographic data from these patients are provided in Supplemental Table 2 (supplemental material available online with this article; https://doi.org/10.1172/jci.insight.121882DS1). Patients with EV-D68 infection showed significantly higher nasopharyngeal IL-17 mRNA levels than patients with RV-A infection (Figure 7).

\section{Discussion}

In 2014, a nationwide outbreak of EV-D68 was associated with severe asthma-like symptoms (9). We sought to develop a mouse model of epidemic EV-D68 infection and determine mechanisms underlying EV-D68induced airway disease. EV-D68 underwent limited viral replication in the lungs of mice, with viral RNA peaking 12 hours after infection. However, infection stimulated airway neutrophilic inflammation, mRNA expression of lung cytokines (TNF- $\alpha$, IL-12b, and IL-17A) and chemokines (CCL2, CXCL1, CXCL2, and CXCL10), and airway hyperresponsiveness, which were suppressed by anti-IL-17A. Neutrophils; mRNA expression of CXCL1, CXCL2, and IL-17; IL-17 protein abundance; and airway responsiveness were significantly higher after EV-D68 infection than after RV-A1B infection. Flow cytometric analysis showed the differential IL-17 production to originate from ILC3s. Finally, in allergen-challenged mice, EV-D68 infection increased BAL eosinophil counts as well as lung mRNA expression of CCL11, IL-5, IL-13, and Muc5AC. Together, these results, which show greater airway responses after EV-D68 than RV-A1B infection and exacerbation of allergic airway disease after EV-D68 infection, are consistent with the clinical picture of severe asthma-like symptoms after EV-D68 infection and provide insight into potential mechanisms.

Recently developed animal models of EV-D68 infection have provided limited information about the pathogenesis of EV-D68-induced airway disease. Nasal inoculation of cotton rats with a recently isolated EV-D68 strain (VANBT/1) showed a limited amount of viral replication, significant induction of pulmonary cytokine mRNA expression (CCL2, CCL5, CXCL1, CXCL10, IL-6, IFN- $\beta$, and IFN- $\gamma$ ), and histologic evidence of peribronchiolitis and alveolitis (32). Nasal infection of ferrets with the EV-D68 Fermon strain increased lung protein abundance of IL-1 $\alpha$, IL-5, IL-8, IL-12, IL-13, and IL-17A as well as alveolar inflammation and hemorrhage (33). These reports did not compare EV-D68-induced airway responses to those induced by RV, nor did they assess changes in airway function or the requirement of various cells or cytokines for EV-D68-induced airway responses.

In the present study, we found that EV-D68-induced airway inflammation and hyperresponsiveness were significantly greater than those induced by RV-A1B. Forty-eight hours after EV-D68 infection, total airway resistance at the maximum dose of methacholine was approximately twice that observed after 
A

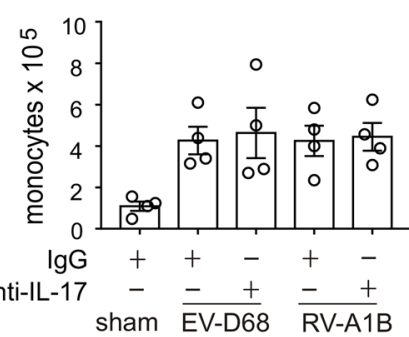

B
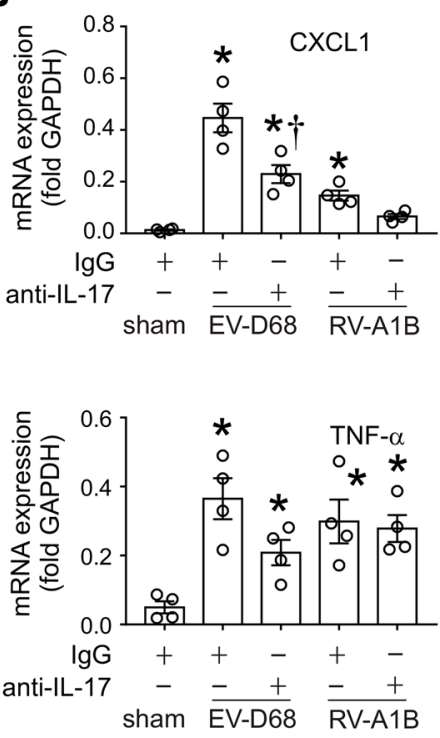
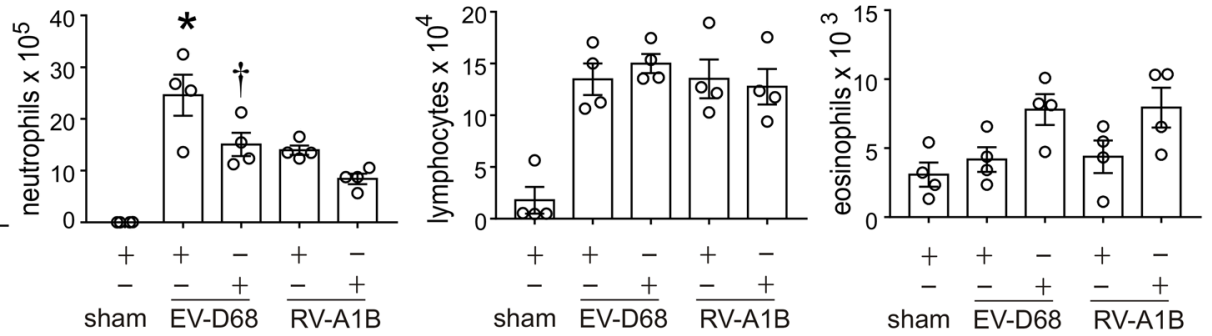
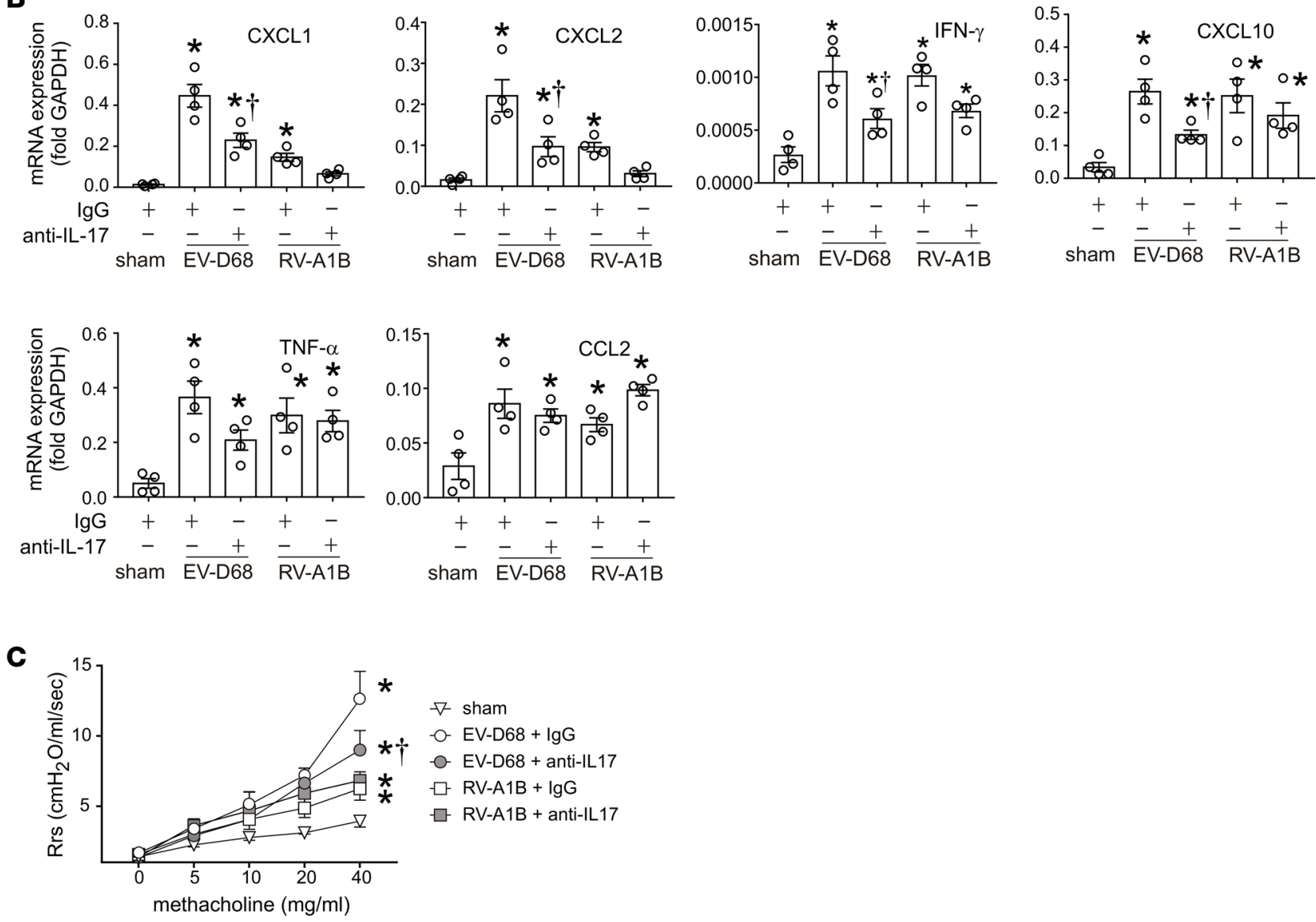

Figure 5. Treatment with anti-IL-17 antibody reduces EV-D68-induced airway responsiveness. Female 8- to 10-week-old BALB/c mice were treated with sham, $5 \times 10^{6} \mathrm{ePFU}$ of EV-D68, or $5 \times 10^{6} \mathrm{PFU}$ of RV-A1B alone or in combination with anti-IL-17 antibody or isotype control. Changes in airway resistance to nebulized increasing doses of methacholine were assessed in tracheotomized mice using a Buxco FinePointe plethysmograph. BAL and mRNA analysis was also carried out in similarly treated mice. (A) BAL cell counts for the indicated groups. (B) qPCR analysis of lung mRNA expression. Data are shown as mean \pm SEM for 4 mice in each group from a single experiment. ${ }^{*} P<0.05$ by ANOVA, compared with RV-1B; $\uparrow P<0.05$ by ANOVA, compared with IgG. (C) Airway responsiveness to increasing doses of methacholine. $n=3-4$ /group from a single experiment. ${ }^{*} P<0.05$ by 2 -way ANOVA, compared with sham; $\uparrow P$ $<0.05$ by 2 -way ANOVA, compared with EV-D68 + IgG.

RV-A1B and comparable to that seen after ovalbumin or HDM sensitization and challenge. Differential airway responsiveness correlated with neutrophilic inflammation and greater mRNA and protein expression of IL-17A. IL-17, in turn, induces production of other proinflammatory cytokines (e.g., IL-1 and TNF- $\alpha$ ), chemokines (e.g., CXCL1, CXCL2, and CCL2), and growth factors (e.g., IL-6, G-CSF, GM-CSF) from tissue structural cells, including fibroblasts, epithelial, and endothelial cells. As a result, the IL-17 response leads to an accumulation of neutrophils at the sites of infection and inflammation (reviewed in ref. 34). Accordingly, EV-D68 infection was associated with significantly higher induction of CXCL1, CXCL2, CCL2, and TNF- $\alpha$. To test for the requirement of IL-17-induced airway responses, mice were treated with a neutralizing antibody against IL-17A. Mice pretreated with anti-IL-17 prior to EV-D68 
A
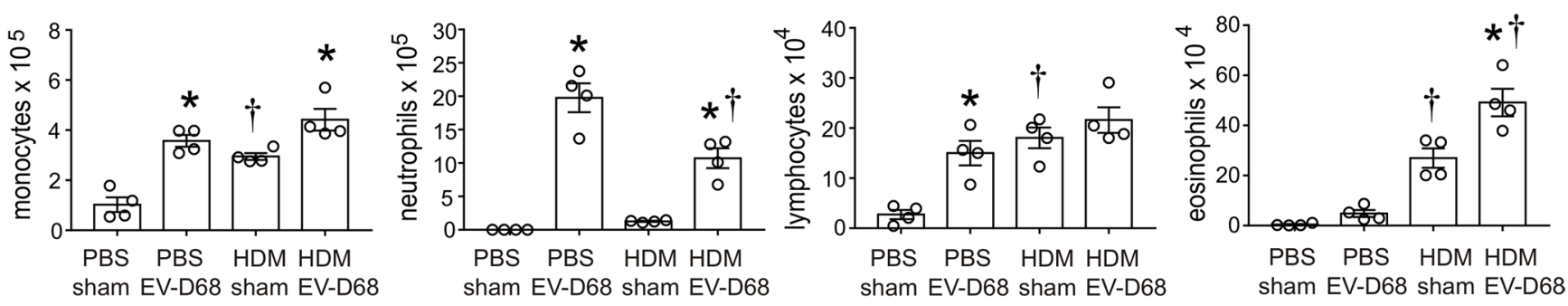

\section{B}
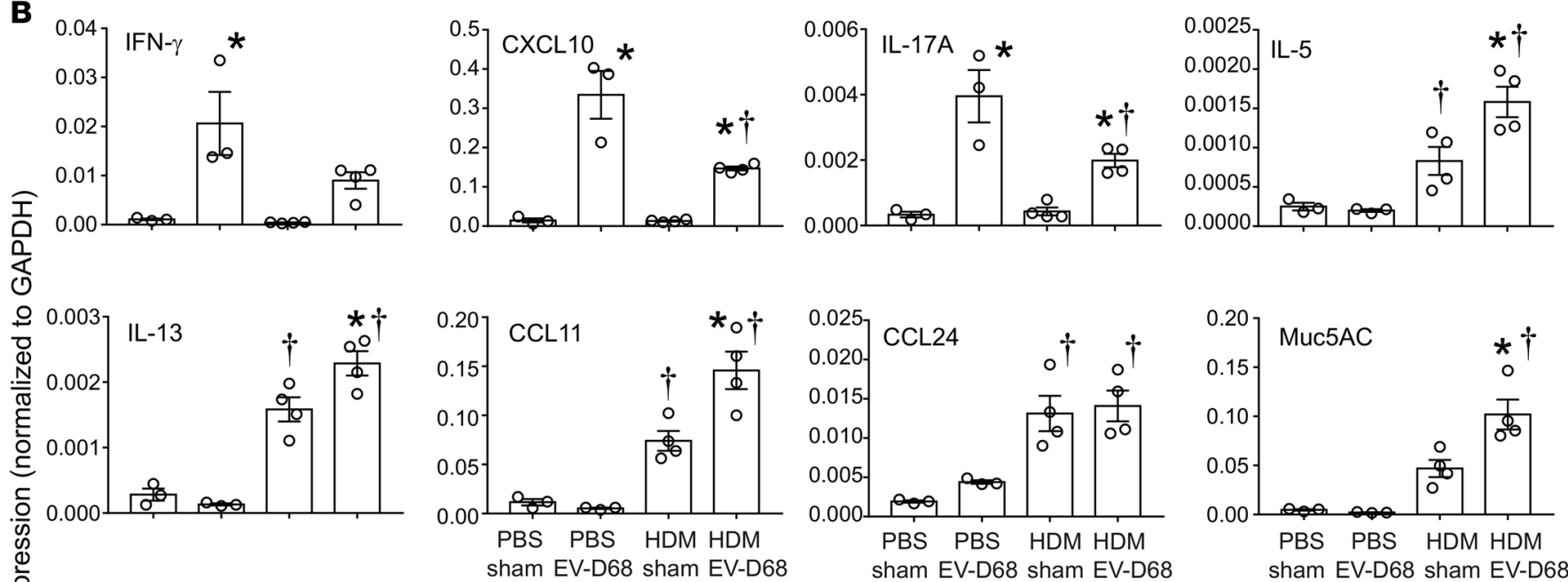
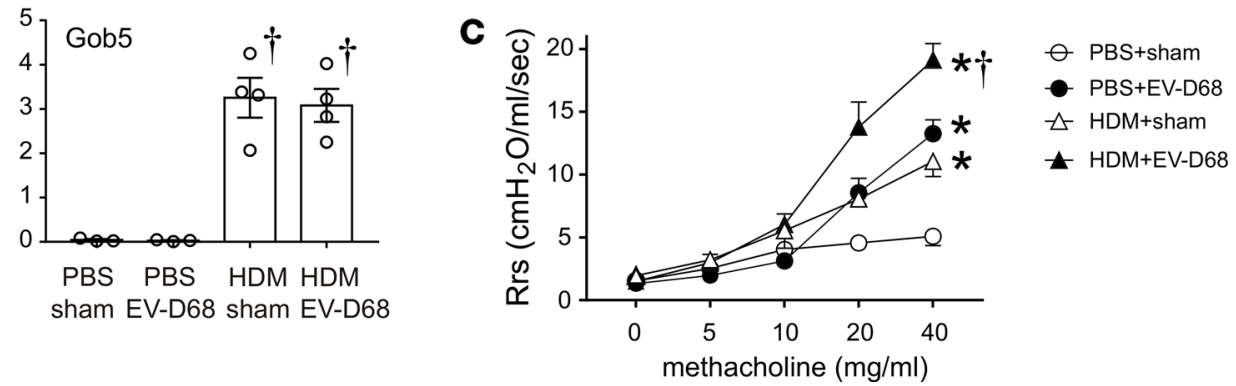

Figure 6. EV-D68 induces type $\mathbf{2}$ cytokines, mucus genes, and greater airway hyperresponsiveness than RV-1B in allergen-challenged mice. Female 8- to 12-week-old BALB/c mice were challenged with house dust mite (HDM) and treated with sham, $5 \times 10^{6}$ ePFU of EV-D68, or $5 \times 10^{6}$ PFU of RV-A1B. Lungs were harvested, and RNA was extracted for QPCR. BAL analysis was also carried out in similarly treated mice. A separate set of mice was similarly treated and anesthetized and endotracheally intubated for measurement of airway responsiveness. (A) BAL cell counts for PBS/sham-, PBS/ EV-D68-, HDM/sham-, and HDM/EV-D68-treated groups. (B) qPCR analysis of the indicated genes for the 4 groups. Data are shown as mean \pm SEM of 3-4 mice/group for a single experiment; ${ }^{*} P<0.05$ by ANOVA, compared with sham, $\uparrow P<0.05$ by ANOVA, compared with PBS. (C) Airway methacholine responsiveness of the indicated treatment groups. $n=3-4$ /group from a single experiment; ${ }^{*} P<0.05$ by 2 -way ANOVA, compared with sham; $† P<0.05$ by 2-way ANOVA, compared with all other groups.

infection showed lower neutrophil infiltration and airway responsiveness compared with mice infected with EV-D68 and pretreated with IgG, indicating that IL-17 is required for neutrophilic inflammation and airway hyperresponsiveness in this context. Reduced airway inflammation and hyperresponsiveness were accompanied by decreased CXCL1 and CXL2 expression, thereby suggesting an effect on downstream effectors. We have shown previously that granulocyte depletion using a monoclonal antibody against Ly6G, an antigen expressed widely on granulocytes, including neutrophils, blocks viral-induced airway hyperresponsiveness (35). Finally, using nasopharyngeal swabs collected in the fall of 2014, we found that IL-17 mRNA expression levels were higher in EV-D68-infected patients than those in those infected with RV-A, suggesting that IL-17 regulates EV-D68 responses in humans.

In addition to Th17 cells, cells of the innate immune system, including $\gamma \delta^{+} \mathrm{T}$ cells, neutrophils, monocytes, and ILC3s, are capable of producing IL-17 (18-20, 22, 25-31, 34, 36-38). EV-D68-infected mice, but 


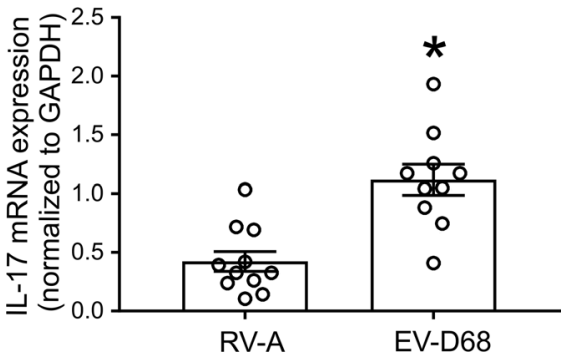

not RV-A1B-infected mice, showed the presence of lineage-ROR $\gamma \mathrm{t}^{+} \mathrm{NKp} 46^{-}$cells. As has been shown previously (25-31), a high percentage of these cells stained positive for IL-17. ILC3s have been reported to be a significant source of IL-17 during various proinflammatory conditions, including bacteria-driven colitis and fibrosis $(29,30)$. In the lung, ILC3s are the predominant source of IL-17A during the early pathogenesis of acute respiratory distress syndrome (39). Flow cytometry also showed significantly more $\gamma \delta \mathrm{T}$ cells in EV-D68-infected mice compared with RV-A1B-infected mice. Like ILC3s, IL-17-producing $\gamma \delta \mathrm{T}$ cells have also been shown to orchestrate the innate immune response to pathogens, including lung infections $(18-21,23,24)$. Together, these results demonstrate for the first time to our knowledge that IL-17-producing ILC3s and $\gamma \delta \mathrm{T}$ cells participate in the immune response to EV-D68 infection.

We examined the effects of HDM sensitization and challenge on EV-D68-induced responses. While EV-D68 infection increased airway neutrophils and lung mRNA expression of IFN- $\gamma$, CXCL10, and IL-17A in HDM-exposed mice, responses were lower than those in EV-D68-infected naive nice, consistent with previous studies showing that allergen sensitization dampens antiviral immunity (reviewed in ref. 40). However, in the context of allergen exposure, EV-D68 infection had synergistic effects on BAL eosinophils and lung IL-5, IL-13, CCL11, and Muc5AC mRNA expression. The precise cell responsible for EV-D68-induced type 2 inflammation in this context was not identified. We have previously shown that RV-A1B infection of allergen-sensitized mice increases type 2 cytokines from alternatively activated macrophages (41). EV-D68 infection of HDM-exposed mice also significantly increased airway responsiveness. These results, which show greater airway responses after EV-D68 infection than RV-A1B infection in both naive and allergic mice, are consistent with the clinical picture of severe asthma-like symptoms after epidemic EV-D68 infection.

We conclude that, in naive mice, EV-D68 induces neutrophilic airway inflammation and hyperresponsiveness, which is dependent on IL-17. Lungs from EV-D68-infected mice showed an increased number

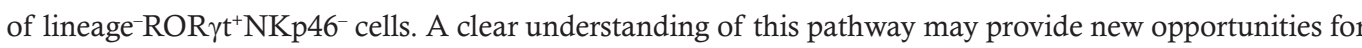
therapeutic intervention in asthma.

\section{Methods}

Generation of EV-D68 and RV-1B. A 2014 isolate of EV-D68 (ATCC) was grown in human rhabdomyosarcoma cells (ATCC). RV-A1B (ATCC), a minor group virus that infects mouse cells (42), was grown in HeLa cells (ATCC). The two viruses were partially purified from infected HeLa cell lysates by means of ultrafiltration with a $100-\mathrm{kDa}$ cutoff filter assay, as described previously (13). Intact virus does not go through the filter and is concentrated. Similarly concentrated and purified HeLa cell lysates were used for sham infection. The infectivity of RV-1B was assessed by plaque assay (43), whereas EV-D68 infectivity was assessed by observing cytopathic effects. Viral RNA was measured in the media by qPCR using specific primers (Supplemental Table 1) and copy number standards.

EV-D68 infection and treatment. 8- to 10-week-old BALB/c mice (The Jackson Laboratory) were inoculated through the intranasal route under Forane anesthesia with EV-D68 (50 $\mu 1$ of $1 \times 10^{8} \mathrm{ePFU} / \mathrm{ml}$ viral stock), RV-1B (50 $\mu 1$ of $1 \times 10^{8} \mathrm{PFU} / \mathrm{ml}$ viral stock), or an equal volume of sham cell lysate.

Real-time quantitative PCR. Lungs were harvested at different points, and RNA was extracted with Trizol (Invitrogen). RNA from nasal washes and lungs was isolated using an RNAeasy kit (Qiagen). cDNA was synthesized from $2 \mu \mathrm{g}$ RNA using a high-capacity cDNA synthesis kit (Applied Biosystems) and subjected to quantitative real-time PCR using specific primers for mRNA (Supplemental Table 1). The level of gene expression for each sample was normalized to GAPDH.

Lung histology and immunofluorescence. Lungs were harvested at different points, fixed with $10 \%$ formaldehyde overnight, and paraffin embedded. Blocks were sectioned at $500-\mu \mathrm{m}$ intervals at a thickness of $5 \mu \mathrm{m}$, 
and each section was deparaffinized, hydrated, and stained. To visualize inflammation, sections were stained with H\&E. Other lung sections were incubated with Alexa Fluor 568-conjugated anti-mouse EV-D68 VP3 (catalog GTX132315, GeneTex). Images were visualized using a ApoTome microscope (Carl Zeiss GmbH).

Flow cytometric analysis. Lungs from sham-, EV-D68-, and RV-A1B-treated mice were perfused with PBS containing EDTA and minced and digested in collagenase IV. Cells were filtered and washed with $\mathrm{RBC}$ lysis buffer, and dead cells were stained with Pac-Blue Live/Dead fixable dead staining dye (Invitrogen). Nonspecific binding was blocked by $1 \%$ fetal bovine serum with $1 \%$ LPS-free bovine serum albumin in DMEM, and $5 \mu \mathrm{g}$ rat anti-mouse CD16/32 (catalog 101302, Biolegend) was added. To identify ILC3s and $\gamma \delta \mathrm{T}$ cells, cells were stained with FITC-conjugated antibodies for lineage markers (CD3e, TCR $\beta$, B220/CD45R, Ter-119, Gr-1/Ly-6G/Ly-6C, CD11b [lineage cocktail, catalog 133301], CD11c [catalog 117306], TCR $\beta$ [catalog 109206], F4/80 [catalog 123108], and FceRI $\alpha$ [catalog 134306] [all from Biolegend]; anti-NKp46-PerCP-Cy5.5 [catalog 137610, Biolegend]; and anti-TCR $\gamma \delta$ [catalog 25-5711-82, eBioscience, Thermo Fisher Scientific]). After staining for cell surface antigens, cells were fixed and permeabilized using Foxp3/Transcription Factor Staining Buffer (eBioscience, Thermo Fisher Scientific) and stained with Alexa Fluor 555-labeled ROR yt (catalog 646502, Biolegend) and Alexa Fluor 647-labeled IL-17 (catalog 506923, Biolegend); Alexa Fluor dyes were from Invitrogen. Cells were subjected to flow cytometry and analyzed on a LSR Fortessa (BD Biosciences). Data were collected using FACSDiva software (BD Biosciences) and analyzed using FlowJo software (Tree Star).

$B A L$. Differential counts of BAL inflammatory cells were performed as described previously (44).

Measurement of airway responsiveness. Mice were anesthetized, intubated, and ventilated with a Buxco FinePointe System. Mice were administered increasing doses of nebulized methacholine to assess airway responsiveness, as previously described (13).

Model of allergic airway disease. 8- to 12-week-old BALB/c mice were sensitized through the intranasal route with $100 \mu \mathrm{g} D$. pteronyssinus HDM extract in $50 \mu 1$ PBS (Greer Labs) by intranasal installation on day 0 and challenged with $10 \mu \mathrm{g}$ HDM on days 11 and 12 . On day 13, mice were inoculated through the intranasal route under Forane anesthesia with EV-D68 (50 $\mu 1$ of $1 \times 10^{8} \mathrm{ePFU} / \mathrm{ml}$ viral stock), RV-A1B (50 $\mu 1$ of $1 \times 10^{8} \mathrm{PFU} / \mathrm{ml}$ viral stock), or an equal volume of sham cell lysate. Forty-eight hours later, mice were analyzed for BAL inflammatory cells, lung mRNA, and airway responsiveness.

$m R N A$ expression from nasopharyngeal swabs of patients with respiratory viral infections. Residual clinical specimens were obtained from the Michigan Medicine Clinical Microbiology Laboratory for characterization of EV-D68. All patients suspected of having a respiratory virus were tested using the FilmArray Respiratory Panel (Biofire Diagnostics) as part of routine care. Residual RV/EV+ samples from September to December 2014 were archived at our request (this test does not distinguish EV from RV infections). RNA was extracted using the QIAamp Viral RNA Mini Kit (Qiagen). EV-D68 was detected by q-RT-PCR (45) and quantified by standard curve; EV-D68- samples collected during peak EV-D68 circulation (September through November 2014) underwent further testing for RV. Assays used for RV detection included q-RTPCR (46) and typing by seminested PCR amplification of the P1-P2 region from gel-purified PCR products (47). Ten EV-D68 samples with $\geq 10^{4}$ copies per $\mathrm{ml}$ were analyzed for IL-17 mRNA by qPCR, and eleven RV-A specimens were frequency-matched by age group were selected for comparison

Statistics. All data are represented as mean \pm SEM. Statistical significance was assessed by unpaired $t$ test or 1-way ANOVA, as appropriate. Group differences were pinpointed by the Student-Newman-Keuls multiple comparison test. $P$ values of less than 0.05 were considered significant.

Study approval. All human studies contained in this publication were approved by the Institutional Review Board of the University of Michigan. Human subjects gave written informed consent in accordance with the ethics principles of the Declaration of Helsinki. All animal usage was approved by the Institutional Animal Care and Use Committee of University of Michigan and followed guidelines set forth in the Principles of Laboratory Animal Care from the National Society for Medical Research.

\section{Author contributions}

CR, ETM, and MBH conceived and designed the research. CR, MH, JKB, JL, TI, QW, JLH, APC, and TLS performed experiments. $\mathrm{CR}$ and $\mathrm{MBH}$ analyzed data, prepared figures, and drafted the manuscript. $\mathrm{MBH}, \mathrm{ETM}$, and WTJ edited and revised the manuscript. CR, MBH, ETM, and WTJ interpreted results of experiments. MBH and ETM approved the final version of the manuscript. 


\section{Acknowledgments}

This work was supported by NIH grants HL134369 and AI120526 (to MBH), NIH grant AI104928 (to WTJ), and "MCubed" grant from the University of Michigan (to MBH, ETM, and TLS).

Address correspondence to: Marc B. Hershenson, Medical Sciences Research Building II, 1150 W. Medical Center Drive, Ann Arbor, Michigan, USA. Phone: 734.936.4200; Email: mhershen@umich.edu.

1. Oberste MS, et al. Enterovirus 68 is associated with respiratory illness and shares biological features with both the enteroviruses and the rhinoviruses. J Gen Virol. 2004;85(Pt 9):2577-2584.

2. Ishiko $\mathrm{H}$, et al. Human rhinovirus 87 identified as human enterovirus 68 by VP4-based molecular diagnosis. Intervirology. 2002;45(3):136-141.

3. Schieble JH, Fox VL, Lennette EH. A probable new human picornavirus associated with respiratory diseases. Am J Epidemiol. 1967;85(2):297-310

4. Centers for Disease Control Prevention (CDC). Clusters of acute respiratory illness associated with human enterovirus 68-Asia, Europe, and United States, 2008-2010. MMWR Morb Mortal Wkly Rep. 2011;60(38):1301-1304.

5. Messacar K, Abzug MJ, Dominguez SR. 2014 outbreak of enterovirus D68 in North America. J Med Virol. 2016;88(5):739-745.

6. Non-Polio Enterovirus: Outbreak and Surveillance Centers for Disease Control and Prevention. https://www.cdc.gov/non-polio-enterovirus/outbreaks-surveillance.html. Accessed August 2, 2018.

7. Maloney JA, Mirsky DM, Messacar K, Dominguez SR, Schreiner T, Stence NV. MRI findings in children with acute flaccid paralysis and cranial nerve dysfunction occurring during the 2014 enterovirus D68 outbreak. AJNR Am J Neuroradiol. 2015;36(2):245-250.

8. Holm-Hansen CC, Midgley SE, Fischer TK. Global emergence of enterovirus D68: a systematic review. Lancet Infect Dis. 2016;16(5):e64-e75.

9. Midgley CM, et al. Severe respiratory illness associated with a nationwide outbreak of enterovirus D68 in the USA (2014): a descriptive epidemiological investigation. Lancet Respir Med. 2015;3(11):879-887.

10. Moss RB. Enterovirus 68 Infection--Association with Asthma. J Allergy Clin Immunol Pract. 2016;4(2):226-228.

11. Foster CB, et al. A comparison of hospitalized children with enterovirus D68 to those with rhinovirus. Pediatr Pulmonol. 2017;52(6):827-832.

12. de Jong MD, et al. Fatal outcome of human influenza A (H5N1) is associated with high viral load and hypercytokinemia. Nat Med. 2006;12(10):1203-1207.

13. Newcomb DC, et al. Human rhinovirus 1B exposure induces phosphatidylinositol 3-kinase-dependent airway inflammation in mice. Am J Respir Crit Care Med. 2008;177(10):1111-1121.

14. Zhou X, Chen Q, Moore J, Kolls JK, Halperin S, Wang J. Critical role of the interleukin-17/interleukin-17 receptor axis in regulating host susceptibility to respiratory infection with Chlamydia species. Infect Immun. 2009;77(11):5059-5070.

15. Mizutani N, Nabe T, Yoshino S. IL-17A promotes the exacerbation of IL-33-induced airway hyperresponsiveness by enhancing neutrophilic inflammation via CXCR2 signaling in mice. J Immunol. 2014;192(4):1372-1384.

16. Yang XO, et al. Regulation of inflammatory responses by IL-17F. J Exp Med. 2008;205(5):1063-1075.

17. Mebratu YA, Tesfaigzi Y. IL-17 Plays a role in respiratory syncytial virus-induced lung inflammation and emphysema in elastase and LPS-injured mice. Am J Respir Cell Mol Biol. 2018;58(6):717-726.

18. Lockhart E, Green AM, Flynn JL. IL-17 production is dominated by gammadelta T cells rather than CD4 T cells during Mycobacterium tuberculosis infection. J Immunol. 2006;177(7):4662-4669.

19. Shibata K, Yamada H, Hara H, Kishihara K, Yoshikai Y. Resident Vdelta1+ gammadelta T cells control early infiltration of neutrophils after Escherichia coli infection via IL-17 production. J Immunol. 2007;178(7):4466-4472.

20. Hamada S, et al. IL-17A produced by gammadelta $\mathrm{T}$ cells plays a critical role in innate immunity against listeria monocytogenes infection in the liver. J Immunol. 2008;181(5):3456-3463.

21. Simonian PL, Roark CL, Born WK, O'Brien RL, Fontenot AP. Gammadelta T cells and Th17 cytokines in hypersensitivity pneumonitis and lung fibrosis. Transl Res. 2009;154(5):222-227.

22. Murdoch JR, Lloyd CM. Resolution of allergic airway inflammation and airway hyperreactivity is mediated by IL-17-producing \{gamma\} \{delta\} T cells. Am J Respir Crit Care Med. 2010;182(4):464-476.

23. Okamoto Yoshida Y, et al. Essential role of IL-17A in the formation of a mycobacterial infection-induced granuloma in the lung. J Immunol. 2010;184(8):4414-4422.

24. Dejima T, et al. Protective role of naturally occurring interleukin-17A-producing $\gamma \delta \mathrm{T}$ cells in the lung at the early stage of systemic candidiasis in mice. Infect Immun. 2011;79(11):4503-4510.

25. Sanos SL, et al. RORgammat and commensal microflora are required for the differentiation of mucosal interleukin 22-producing NKp46+ cells. Nat Immunol. 2009;10(1):83-91.

26. Satoh-Takayama N, et al. Microbial flora drives interleukin 22 production in intestinal NKp46+ cells that provide innate mucosal immune defense. Immunity. 2008;29(6):958-970.

27. Luci C, et al. Influence of the transcription factor RORgammat on the development of NKp46+ cell populations in gut and skin. Nat Immunol. 2009;10(1):75-82.

28. Takatori H, et al. Lymphoid tissue inducer-like cells are an innate source of IL-17 and IL-22. J Exp Med. 2009;206(1):35-41.

29. Buonocore S, et al. Innate lymphoid cells drive interleukin-23-dependent innate intestinal pathology. Nature. 2010;464(7293):1371-1375.

30. Lo BC, et al. The orphan nuclear receptor ROR alpha and group 3 innate lymphoid cells drive fibrosis in a mouse model of Crohn's disease. Sci Immunol. 2016;1(3): eaaf8864.

31. Klose CS, et al. A T-bet gradient controls the fate and function of CCR6-ROR $\gamma \mathrm{t}+$ innate lymphoid cells. Nature. 
2013;494(7436):261-265.

32. Patel MC, et al. Enterovirus D-68 infection, prophylaxis, and vaccination in a novel permissive animal model, the cotton rat (Sigmodon hispidus). PLoS One. 2016;11(11):e0166336

33. Zheng HW, et al. Nasal infection of enterovirus D68 leading to lower respiratory tract pathogenesis in ferrets (Mustela putorius furo). Viruses. 2017;9(5):E104

34. Kolls JK, Lindén A. Interleukin-17 family members and inflammation. Immunity. 2004;21(4):467-476.

35. Nagarkar DR, et al. CXCR2 is required for neutrophilic airway inflammation and hyperresponsiveness in a mouse model of human rhinovirus infection. J Immunol. 2009;183(10):6698-6707.

36. Ferretti S, Bonneau O, Dubois GR, Jones CE, Trifilieff A. IL-17, produced by lymphocytes and neutrophils, is necessary for lipopolysaccharide-induced airway neutrophilia: IL-15 as a possible trigger. J Immunol. 2003;170(4):2106-2112.

37. Song C, et al. IL-17-producing alveolar macrophages mediate allergic lung inflammation related to asthma. J Immunol. 2008;181(9):6117-6124.

38. Cupedo T, et al. Human fetal lymphoid tissue-inducer cells are interleukin 17-producing precursors to RORC+ CD127+ natural killer-like cells. Nat Immunol. 2009;10(1):66-74

39. Muir R, et al. Innate lymphoid cells are the predominant source of IL-17A during the early pathogenesis of acute respiratory distress syndrome. Am J Respir Crit Care Med. 2016;193(4):407-416.

40. Rowe RK, Gill MA. Effects of allergic sensitization on antiviral immunity: allergen, virus, and host cell mechanisms. Curr Allergy Asthma Rep. 2017;17(2):9.

41. Nagarkar DR, et al. Rhinovirus infection of allergen-sensitized and -challenged mice induces eotaxin release from functionally polarized macrophages. J Immunol. 2010;185(4):2525-2535.

42. Tuthill TJ, et al. Mouse respiratory epithelial cells support efficient replication of human rhinovirus. J Gen Virol. 2003;84(Pt 10):2829-2836.

43. Martin S, Casasnovas JM, Staunton DE, Springer TA. Efficient neutralization and disruption of rhinovirus by chimeric ICAM1/immunoglobulin molecules. J Virol. 1993;67(6):3561-3568.

44. Tsai WC, et al. Azithromycin blocks neutrophil recruitment in Pseudomonas endobronchial infection. Am J Respir Crit Care Med. 2004;170(12):1331-1339.

45. Enterovirus D68 2014 Real-Time RT-PCR Assay. Centers for Disease Control and Prevention. http://www.fda.gov/downloads/ MedicalDevices/Safety/EmergencySituations/UCM446784.pdf. Accessed August 2, 2018.

46. Contoli M, et al. Role of deficient type III interferon-lambda production in asthma exacerbations. Nat Med. 2006;12(9):1023-1026.

47. Lee WM, et al. A diverse group of previously unrecognized human rhinoviruses are common causes of respiratory illnesses in infants. PLoS One. 2007;2(10):e966 